\title{
A Novel Rat Model of Embolic Cerebral Ischemia Using a Microcatheter and Zirconia Ball Under Fluoroscopic Guidance
}

Teppei Komatsu ( $\square$ teppeinoieni.coicoi@jikei.ac.jp )

Jikei University School of Medicine

\section{Hiroki Ohta}

Jikei University School of Medicine

Haruhiko Motegi

Jikei University School of Medicine

Junichi Hata

Jikei University School of Medicine

Koshiro Terawaki

Tokyo Metropolitan University

Makoto Koizumi

Jikei University School of Medicine

Kanako Muta

University of Tokyo

Hirotaka James Okano

Jikei University School of Medicine

Yasuyuki Iguchi

Jikei University School of Medicine

\section{Research Article}

Keywords: Ischemic stroke, Animal model, Rat, Middle cerebral artery occlusion

Posted Date: March 11th, 2021

DOI: https://doi.org/10.21203/rs.3.rs-264121/v1

License: (a) (i) This work is licensed under a Creative Commons Attribution 4.0 International License. Read Full License 


\section{Abstract}

Background and Purpose: The failure of neuroprotective treatment-related clinical trials may be partially caused by unestablished animal models. Existing animal models are less likely to provide occlusion confined to the middle cerebral artery (MCA), making transarterial intervention difficult. We aimed to develop a novel focal stroke model using a microcatheter and zirconium dioxide that is non-magnetic under fluoroscopic guidance, which can monitor MCA occlusion and can improve hemorrhagic complications.

Methods: Using male Sprague Dawley rats $(n=8)$, a microcatheter was navigated from the caudal ventral artery to the left internal carotid artery using digital subtraction angiography to establish local occlusion.

Results: All rat cerebral angiographies were successful. No rats had hemorrhagic complications. Six (75\%) rats underwent occlusion of the MCA bifurcation by zirconium dioxide. Accidentally, the left posterior cerebral artery was failure embolized in 2 rats (25\%) via the posterior communicating artery. The median operating time was $8.5 \mathrm{~min}$. Moreover, $2 \%$ 2,3,5-triphenyl tetrazolium chloride staining showed that the median infarct volume $\left(\mathrm{mm}^{3}\right)$ was 280 (interquartile range; 267-333) 24 hours after the left MCA bifurcation occlusion.

Conclusions: We present a novel rat model for focal stroke using a microcatheter and zirconium dioxide which does not affect the MRI. The model is predictable and reproducible, which is well confined within the territory supplied by the MCA. Fluoroscopy was able to identify which the MCA occlusion and model success while creating the model. It permitted exclusion of animals with complications from the experiment.

\section{Introduction}

Stroke is the second most common cause of death and the third most common cause of disabilityadjusted life years lost worldwide ${ }^{1}$. In most patients, ischemic stroke results from occlusion of the middle cerebral artery (MCA). As such, rodent models have been developed to mimic human focal ischemic stroke ${ }^{2}$. The most widely used rat model for focal cerebral ischemic stroke involves an intra-arterial suture occlusion of the MCA (MCAO), in which a nylon thread is blindly inserted from the external carotid artery $(E C A)^{3,4}$. The main limitation of this model is that blood flow to the posterior communicating artery (PcomA) and branches of the internal carotid artery (ICA) are obstructed, resulting in large and variable infarctions of the cortical and subcortical areas ${ }^{5-11}$. Besides, the procedure is very invasive because the ECA is ligated after the neck incision. Ligation of the ECA results in ischemic necrosis of the mastication and hypopharyngeal muscles, which negatively affects behavioral testing outcomes ${ }^{12,13}$. There are additional models for inducing stroke, all with merits and limitations (Table 1) 3,4,14-19.

There has been growing interest regarding the use of regenerative medicine for sequelae of cerebral infarctions, and clinical trials on intravenous administration of bone marrow mesenchymal cells have commenced. However, intravenous administration may result in the medicine being taken into organs other 
than the target, such as the lungs, thereby reducing the therapeutic effect. Therefore, it is necessary to urgently develop a highly reproducible animal model that can verify intra-arterial cell administration for cerebral infarctions.

Here, we present a highly selective rat MCAO model by percutaneous caudal arterial puncture using a microcatheter and zirconia ball under fluoroscopic guidance. The model is predictable and reproducible, which is well confined within the territory supplied by the MCA, and the surgical procedures can be typically completed within $10 \mathrm{~min}$. Furthermore, minimally invasive and highly reproducible procedure lead to a "reduction" in the suffering and "refinement" of the welfare of laboratory animals.

\section{Materials And Methods}

The authors declare that all supporting data are available within the article.

\section{Animal model and protocol}

Male normotensive adult Sprague Dawley rats ( $n=8 ; 11-27$ weeks of age; $366-587 \mathrm{~g}$ on the day of surgery) obtained from Nihon SLC (Japan SLC, Inc. Shizuoka, Japan) were housed in polycarbonate cages under temperature-controlled conditions (temperature: $24-25^{\circ} \mathrm{C}$; relative humidity: $50-60 \%$ ) with a 12 -h light-dark cycle. All rats had free access to water and pelleted food (CE-2, CLEA Japan, Inc., Gifu, Tokyo, Japan). Our study was approved by the Institutional Animal Care and Use Committee of the Jikei University School of Medicine (protocol number: 2016-105). All procedures were conducted according to the Fundamental Guidelines for Proper Conduct of Animal Experiments and Related Activities in Academic Research Institutions issued by the Japanese Ministry of Education, Culture, Sports, Science, and Technology. This study doesn't need the control group because of making stroke model study. Anesthesia was maintained with 1-3\% isoflurane through a vaporizer for small experimental animals and a facial mask. Body temperature was maintained during surgery at $36-37.5^{\circ} \mathrm{C}$ using a multi-panel heater (Vivaria, Osaka, Japan). The animal's body, including the entire tail, must be kept warm, as previously reported. ${ }^{20}$ No preparation, such as shaving, disinfection, or antibiotics, is required.

Major arteries of rat with microcatheter and zirconia ball and a simplified operation scheme are shown in Fig.1. The polyamide microcatheter (inner diameter (ID) $0.42 \mathrm{~mm}$, outer diameter (OD) $0.55 \mathrm{~mm}$, Kaneko Cord, Tokyo, Japan) was first flushed with heparinized physiological saline. The rat was placed in a supine position with a stretched tail. Indwelling needle (venous indwelling needle for human, 22 gauge, SROT2225C, TERUMO, Tokyo) was inserted through the ventral midline artery approximately $5 \mathrm{~cm}$ from the root of the tail of the rat (Fig. 2A). After confirmation of arterial blood backflow from the indwelling needle, a microcatheter and wire (OD 0.4mm, FGW16-AG18S30, Toray Medical, Tokyo, Japan) were inserted as long as no resistance was felt. Upon resistance, the insertion of the microcatheter was stopped immediately, and the position of the microcatheter was confirmed using fluoroscopic imaging. The wire and microcatheter were guided from the caudal ventral artery to the abdominal aorta (Fig. 2B), the aortic arch, and the left common carotid artery (CCA) (Fig. 2C) using fluoroscopic imaging. Then, the wire was carefully removed so as not to change the position of the microcatheter. The contrast media (lohexol, 
Daiichi Sankyo, Tokyo, Japan) at half concentration was rapidly injected from the microcatheter. Then, left CCA angiography was performed, in which $0.1 \mathrm{ml}$ of the contrast media was injected using a digital subtraction angiography unit (Artis Zee, Siemens, Germany; Fig. 2D). The bifurcation of the ICA branch was visualized by tilting the $\mathrm{C}$-arm at an $\mathrm{LAO}$ angle of $80^{\circ}$ (Fig. 2D). The contrast images were overlaid for improving visualization, and the microcatheter was guided to the branch of the left ICA. Left ICA selective angiography could then detect the left anterior cerebral artery (ACA), MCA, and PcomA (Fig. 3A). Then, one zirconia ball (zirconium dioxide, $0.4 \mathrm{~mm}$ in diameter; Nikkato, Tokyo, Japan. Fig. 4A) was advanced in the microcatheter by slow injection of approximately $0.1 \mathrm{ml}$ of heparinized physiological saline (Supplemental File - Videos 1 and 2), and the ACA-MCA bifurcation was selectively embolized (Figs. 3B, 4B). The zirconium dioxide does not affect the MRI because of non-magnetic material. The high visibility of zirconia ball during fluoroscopy was found to be comparable to that of wire. The microcatheter was inserted into the right CCA and contrasted to confirm that the left ACA had retrograde blood flow from the right ACA and only obstructed the left MCA in the frontal view (LAO angle $0^{\circ}$ ) (Fig. $3 \mathrm{C}$ ). The time from the puncture to model creation was measured. The plastic outer indwelling needle was fixed with sealing film (Parafilm, Bemis, United States of America) and gaffer tape and was not removed until 24 hours after the embolization. Then, cerebral angiography was performed to confirm whether spontaneous recanalization had occurred. No postoperative pain relief was required.

\section{Cerebral angiography 24 hours after the creation of the stroke model}

Cerebral angiography was performed 24 hours after the creation of the stroke model to determine whether spontaneous recanalization had occurred. Cerebral angiography could be performed in the same way as when the model was created without re-puncture by using the plastic outer indwelling needle that was left behind.

\section{Magnetic resonance imaging (MRI) analysis}

MRI was performed using a 9.4-T BioSpec 94/20 (Biospin GmbH, Ettlingen, Germany) device and a transmitting coil with a 72-mm inner diameter and a rat brain size 4 channel receiver coil immediately before the rats were euthanized. T2-weighted imaging and MR angiography were performed using the time of flight theory. T2-weighted imaging was performed using the rapid acquisition with a relaxation enhancement pulse sequence ${ }^{21}$. The following parameters were set: repetition time: $5000 \mathrm{~ms}$, effective echo time: $42.0 \mathrm{~ms}$, flip angle: $90^{\circ}$, RARE factor: 8 , field of view: $20 \times 20 \mathrm{~mm}^{2}$, matrix size: $200 \times 200$, image resolution: $100 \times 100 \mu \mathrm{m}$, and slice thickness: $800 \mu \mathrm{m}$. The total T2-weighted imaging scan time was 6 minutes and 15 seconds. MR angiography was performed using a three-dimensional fast low angle shot pulse sequence ${ }^{22}$. The following parameters were set to achieve the time of flight effect: repetition time: 14 ms, echo time: $2.28 \mathrm{~ms}$, flip angle: $8^{\circ}$, number of average: 6 , field of view: $20 \times 18 \times 22 \mathrm{~mm}^{2}$, matrix size: $166 \times 148 \times 180$, and image resolution: $122 \times 122 \times 122 \mu \mathrm{m}$. The total scan time of the three-dimensional fast low angle shot pulse sequence was 36 minutes 20 seconds. MR angiography was reconstructed using the maximum intensity projection method in ParaVision version 6.0.1 software (Bruker, Ettlingen, Germany). To acquire MRI data, the rats were scanned in the prone position on an imaging stretcher and administered a 
mixture of air and 1.5-3.0 \% concentrated isoflurane (Abbott Laboratories, Abbott Park, IL, USA). Respiration was regularly monitored during the scanning to manage the animal's physical condition.

\section{Postmortem analysis}

Twenty-four hours after embolization, the animals were deeply anesthetized and euthanized. The brains were removed and were sectioned coronally into 6 slices (thickness, $2 \mathrm{~mm}$ ) from the olfactory bulb to the cerebellum and then stained with 2\% 2,3,5-triphenyl tetrazolium chloride (TTC) for 20 minutes. Using image analysis software (ImageJ, version 1.53a), the non-infarcted area of the ipsilateral hemispheres and the areas of the contralateral hemisphere were calculated on the rostral side and caudal side, respectively.

Infarct volume on each section $\left(\mathrm{mm}^{3}\right)=$ (areas of the contralateral hemisphere - the non-infarcted area of the ipsilateral hemisphere) on the rostral side $\left(\mathrm{mm}^{2}\right) \times 1(\mathrm{~mm})+$ (areas of the contralateral hemisphere the non-infarcted area of the ipsilateral hemisphere) on the caudal side $\left(\mathrm{mm}^{2}\right) \times 1(\mathrm{~mm})$

The sum of each section volume is considered to be the total volume of infarction ${ }^{23,24}$. A postmortem analysis was performed by one researcher (H.M.), who was blinded from details of the surgical procedures.

\section{Results}

All rat cerebral angiographies were successful. There no exclusions. No rats had hemorrhagic complications including a subarachnoid hemorrhage (SAH). Six (75\%) rats underwent occlusion of the ACA-MCA bifurcation by zirconia ball (Figs. 3B, 3C, 4B, Supplemental File - Video 1). Accidentally, the left posterior cerebral artery was failure embolized in 2 rats $(25 \%)$ through the PcomA (Fig. 3D, Supplemental File - Video 2). Fluoroscopy was able to identify which the MCAO and model success while creating the model. The median operating time was $8.5 \mathrm{~min}$ (interquartile range [IQR]; 6-14.25 min). The maximum contrast material dose was up to $1 \mathrm{ml}$, and the intraoperative blood loss was less than $1 \mathrm{ml}$. No cases of spontaneous recanalization 24 hours after the embolization occurred. No tails had ischemia because the 2 lateral caudal arteries took over the supply through numerous anastomoses. There were no deaths within the 24 hours before the postmortem analysis. The zirconia ball did not affect the MRI (Fig. 5). Furthermore, $2 \%$ TTC staining showed that the median infarct volumes $\left(\mathrm{mm}^{3}\right)$ were 280 (IQR; 267-333) 24 hours after the left MCA bifurcation occlusion (Fig. 6A). Twenty-four hours after the left PcomA occlusion, the stroke lesion volume was $251 \mathrm{~mm}^{3}$ and $446 \mathrm{~mm}^{3}$ (Fig. 6B). There was no infarction of the thalamus and hypothalamus in MCAO model.

\section{Discussion}

We have presented a novel zirconia MCAO rat model that is minimally invasive and highly reproducible. To the best of our knowledge, this is the first study to present a highly selective MCAO model by percutaneous caudal arterial puncture using zirconia ball and microcatheter under fluoroscopic guidance. Fluoroscopy was able to identify which the MCAO and model success while creating the model. It permitted exclusion of 
animals with complications from the experiment. The advantages of the zirconia MCAO rat model for experimental studies are discussed below and are summarized in Table 1.

First, this novel model can create an infarct only in the MCA territory without ischemia of the ECA, the PcomA, and branches of the ICA, and can also identify the site of vessel occlusion intraoperatively. All existing models of MCAO except caudal artery approach share the same disadvantages. Ligation of the ECA and insertion of the suture occludes the entire course of the ICA, leading to obstruction of the ECA, the PcomA, and branches of the ICA, such as the hypothalamic and anterior choroidal arteries. Ligation of the ECA results in ischemic necrosis of the mastication and hypopharyngeal muscles, which negatively affects behavioral testing outcomes ${ }^{12}$. The PcomA and branches of the ICA are the main arteries to the thalamus, hypothalamus, hippocampus, and substantia nigra ${ }^{25-27}$. As such, because these important areas are infarcted, the animal have a variable level of increased body temperature, disturbed water homeostasis, severe paresis, and high postoperative mortality, which altogether can result in bias in experimental studies $^{5-11}$. The macrosphere and suture models lead to reduced blood flow in the ECA and anterior choroidal artery ${ }^{14,15}$. The zirconia MCAO model can reduce inter-animal variability and mortality by selectively causing ischemia only in the MCA blood flow area.

Second, the zirconia MCAO model is minimally invasive and safe as it only requires a caudal artery puncture and does not require a craniotomy and cervical incision (Table 1). Moreover, in our study, there was not a single case of hemorrhagic complications. SAH occurred in $8 \%-30 \%$ of animals in the intraluminal suture MCAO model (Table 1). ${ }^{16-18} \mathrm{SAH}$ can lead to serious complications such as vasospasm and intracerebral hemorrhage, and thus can confound the results of experimental stroke studies. If SAH occurs, the zirconia MCAO model can detect SAH while creating the model using fluoroscopy. Minimally invasive and safe methods can prevent the possible loss of the experimental animal, and lead to a "reduction" in the suffering and "refinement" of the welfare of laboratory animals. ${ }^{28}$

Third, the zirconia MCAO model uses fluoroscopy, which means that vessel occlusion and model success can be checked for in real-time while creating the model. This is particularly important for treatment intervention studies using animal models because it avoids performing an intervention with failed models.

Finally, by placing a plastic outer indwelling needle in the caudal artery, cerebral angiography can be repeated. This method can also be used for arterial drug administration, which can be repeated in a highly selective and direct manner to the target artery. Multiple doses of the drug or cells can be administered to the target organ at the time of therapeutic intervention, thereby enabling the establishment of more sophisticated and complex therapeutic intervention studies. Endovascular therapy is already commonly used in humans and is easy to apply clinically.

Our study has several limitations. First, the model failure rate was $25 \%$ due to the occlusion of PcomA instead of MCA. Second, left ACA blood flow was dependent on the right ACA instead of the left ICA. However, no infarcts in the ACA region appeared, so this does not appear to be an issue. Third, some physiological data, such as blood gases and animal body temperatures, were not measured. Finally, the model cannot induce reperfusion. Any of the current preclinical model of stroke including the current one in 
this study has caveats. We think multidirectional approach using different preclinical models can help to better understand the pathophysiology of stroke.

\section{Conclusions}

In conclusion, the zirconia MCAO model presented here has several advantages. It is minimally invasive, and fluoroscopic guidance allows for the selective endovascular occlusion of the MCA. The technique also avoids ischemia of the ECA, anterior choroidal artery, hypothalamic artery, and PcomA. This is important because the confounding effects of tissue damage outside the MCA territory in the MCAO model are considerable. From an ethical standpoint, this model significantly limits the suffering caused by complications secondary to large, unspecific infarct lesions, surgical exploration of the neck, and ligation of the ECA. The microcatheter that carries the microwire can also serve as a working channel wherein therapeutic agents may be administered in a targeted fashion. We hope that this approach will be carried out by many researchers, leading to a "reduction" in the suffering and "refinement" of the welfare of laboratory animals. The development of the zirconia MCAO model also helps reduce costs associated with investing in unnecessary clinical trials, the death of laboratory animals, and ineffective drug discovery.

\section{Declarations}

Acknowledgements: None.

Ethics: Our study was approved by the Institutional Animal Care and Use Committee of the Jikei University School of Medicine (protocol number: 2016-105).

\section{Acknowledgments}

We would like to thank the following staff members for their contributions to this study: Yasuo Kimura, Hazuki Sato, Saori Ogatsu, Yuki Minowa (Laboratory Animal Facilities, the Jikei University School of Medicine).

Sources of funding: This work was supported by MEXT KAKENHI Grant Number 17K18130 (to Teppei Komatsu), $18 \mathrm{~K} 16404$ (to Hiroki Ohta), the Jikei University Research Fund (to Teppei Komatsu), and The Uehara Memorial Foundation (to Teppei Komatsu).

Conflicts of interest: The authors of this manuscript declare no relationships with any companies, whose products or services may be related to the subject matter of the article.

Disclosures: None

Author contributions: T.K., H.O. wrote the main manuscript, H.M. performed the postmortem analysis and M.K., K.M. assisted in animal care. J.H., K.T. performed the MRI analysis. H.O., Y.I. helped conduct the literature review and amended the manuscript. All authors have reviewed the final manuscript.

Significance Statement: 
The model is predictable and reproducible, which is confined within the territory supplied by the MCA.

\section{References}

1. Feigin, V. L. et al. Global and regional burden of stroke during 1990-2010: findings from the Global Burden of Disease Study 2010. Lancet. 383, 245-254 https://doi.org/10.1016/s0140-6736(13)619534 (2014).

2. Durukan, A. \& Tatlisumak, T. Acute ischemic stroke: overview of major experimental rodent models, pathophysiology, and therapy of focal cerebral ischemia. Pharmacol Biochem Behav. 87, 179-197 https://doi.org/10.1016/j.pbb.2007.04.015 (2007).

3. Koizumi, J., Yoshida, Y., Nakazawa, T. \& Ooneda, G. Experimental studies of ischemic brain edema. â... : A new experimental model of cerebral embolism in rats in which recirculation can be introduced in the ischemic area. Jpn J Stroke. 8, 1-8 https://doi.org/10.3995/jstroke.8.1 (1986).

4. Longa, E. Z., Weinstein, P. R., Carlson, S. \& Cummins, R. Reversible middle cerebral artery occlusion without craniectomy in rats. Stroke. 20, 84-91 https://doi.org/10.1161/01.str.20.1.84 (1989).

5. Kanemitsu, H. et al. Differences in the extent of primary ischemic damage between middle cerebral artery coagulation and intraluminal occlusion models. J Cereb Blood Flow Metab. 22, 1196-1204 https://doi.org/10.1097/01.wcb.0000037992.07114.95 (2002).

6. Tamura, A., Graham, D. I., McCulloch, J. \& Teasdale, G. M. Focal cerebral ischaemia in the rat: 1. Description of technique and early neuropathological consequences following middle cerebral artery occlusion. J Cereb Blood Flow Metab. 1, 53-60 https://doi.org/10.1038/jcbfm.1981.6 (1981).

7. Chen, S. T., Hsu, C. Y., Hogan, E. L., Maricq, H. \& Balentine, J. D. A model of focal ischemic stroke in the rat: reproducible extensive cortical infarction. Stroke. 17, 738-743 https://doi.org/10.1161/01.str.17.4.738 (1986).

8. Buchan, A. M., Xue, D. \& Slivka, A. A new model of temporary focal neocortical ischemia in the rat. Stroke. 23, 273-279 https://doi.org/10.1161/01.str.23.2.273 (1992).

9. Li, F., Omae, T. \& Fisher, M. Spontaneous hyperthermia and its mechanism in the intraluminal suture middle cerebral artery occlusion model of rats. Stroke. 30, 2464-2470 discussion $2470-2461$ https://doi.org/10.1161/01.str.30.11.2464 (1999).

10. Fisher, M. \& Tatlisumak, T. Use of animal models has not contributed to development of acute stroke therapies: con. Stroke. 36, 2324-2325 https://doi.org/10.1161/01.STR.0000179039.76922.e8 (2005).

11. Garcia, J. H., Liu, K. F., Ye, Z. R. \& Gutierrez, J. A. Incomplete infarct and delayed neuronal death after transient middle cerebral artery occlusion in rats. Stroke. 28, 2303-2309 discussion 2310 https://doi.org/10.1161/01.str.28.11.2303 (1997).

12. Dittmar, M., Spruss, T., Schuierer, G. \& Horn, M. External carotid artery territory ischemia impairs outcome in the endovascular filament model of middle cerebral artery occlusion in rats. Stroke. 34, 2252-2257 https://doi.org/10.1161/01.str.0000083625.54851.9a (2003).

13. Trueman, R. C. et al. A Critical Re-Examination of the Intraluminal Filament MCAO Model: Impact of External Carotid Artery Transection. Trans/ Stroke Res. 2, 651-661 https://doi.org/10.1007/s12975- 
011-0102-4 (2011).

14. Gerriets, T. et al. The macrosphere model: evaluation of a new stroke model for permanent middle cerebral artery occlusion in rats. J Neurosci Methods. 122, 201-211 https://doi.org/10.1016/s01650270(02)00322-9 (2003).

15. Gerriets, T. et al. Complications and pitfalls in rat stroke models for middle cerebral artery occlusion: a comparison between the suture and the macrosphere model using magnetic resonance angiography. Stroke. 35, 2372-2377 https://doi.org/10.1161/01.STR.0000142134.37512.a7 (2004).

16. Fluri, F., Schuhmann, M. K. \& Kleinschnitz, C. Animal models of ischemic stroke and their application in clinical research. Drug Des Devel Ther. 9, 3445-3454 https://doi.org/10.2147/dddt.s56071 (2015).

17. Schmid-Elsaesser, R., Zausinger, S., Hungerhuber, E., Baethmann, A. \& Reulen, H. J. A critical reevaluation of the intraluminal thread model of focal cerebral ischemia: evidence of inadvertent premature reperfusion and subarachnoid hemorrhage in rats by laser-Doppler flowmetry. Stroke. 29, 2162-2170 https://doi.org/10.1161/01.str.29.10.2162 (1998).

18. Liu, S. et al. J Exp Stroke Trans/ Med. 2, 2-27 https://doi.org/10.6030/1939-067x-2.2.2 (2009).

19. Zhang, R. L., Chopp, M., Zhang, Z. G., Jiang, Q. \& Ewing, J. R. A rat model of focal embolic cerebral ischemia. Brain Res. 766, 83-92 https://doi.org/10.1016/S0006-8993(97)00580-5 (1997).

20. Ohta, H., Ohki, T., Kanaoka, Y., Koizumi, M. \& Okano, H. J. Pitfalls of invasive blood pressure monitoring using the caudal ventral artery in rats. Sci Rep. 7, 41907 https://doi.org/10.1038/srep41907 (2017).

21. Hennig, J., Nauerth, A. \& Friedburg, H. RARE imaging: a fast imaging method for clinical MR. Magn Reson Med. 3, 823-833 https://doi.org/10.1002/mrm.1910030602 (1986).

22. Haase, A., Frahm, J., Matthaei, D., Hanicke, W. \& Merboldt, K. D. FLASH imaging: rapid NMR imaging using low flip-angle pulses 1986. J Magn Reson 213, 533-541, doi:10.1016/j.jmr.2011.09.021 (2011).

23. Swanson, R. A. et al. A semiautomated method for measuring brain infarct volume. J Cereb Blood Flow Metab. 10, 290-293 https://doi.org/10.1038/jcbfm.1990.47 (1990).

24. Zhang, P. et al. Early exercise improves cerebral blood flow through increased angiogenesis in experimental stroke rat model. J Neuroeng Rehabil. 10, 43 https://doi.org/10.1186/1743-0003-10-43 (2013).

25. Brown, J. O. The morphology of circulus arteriosus cerebri in rats. Anat Rec. 156, 99-106 https://doi.org/10.1002/ar.1091560112 (1966).

26. Coyle, P. Arterial patterns of the rat rhinencephalon and related structures. Exp Neurol. 49, 671-690 https://doi.org/10.1016/0014-4886(75)90051-5 (1975).

27. McAuley, M. A. Rodent models of focal ischemia. Cerebrovasc Brain Metab Rev. 7, 153-180 (1995).

28. Carvalho, C., Gaspar, A., Knight, A. \& Vicente, L. Ethical and Scientific Pitfalls Concerning Laboratory Research with Non-Human Primates, and Possible Solutions. Animals (Basel). 9, https://doi.org/10.3390/ani9010012 (2018).

\section{Tables}


Table 1. Comparison between the most prevalent rodent stroke models and the model in the current study

\begin{tabular}{|lllll|}
\hline & $\begin{array}{l}\text { Suture } \\
\text { model }\end{array}$ & $\begin{array}{l}\text { Thromboembolic } \\
\text { model }^{19}\end{array}$ & $\begin{array}{l}\text { Macrosphere } \\
\text { model }^{14,15}\end{array}$ & $\begin{array}{l}\text { Current } \\
\text { model }\end{array}$ \\
\hline Procedure & Incision & Incision & Incision & Puncture \\
\hline Approach site & Neck & Neck & Neck & Tail \\
\hline Affected ischemic area & MCA/ECA/AChA/ & MCA/ECA & MCA/ECA/AChA & MCA \\
\hline $\begin{array}{l}\text { Identify the vessel } \\
\text { Occlusion while creating } \\
\text { the model }\end{array}$ & Impossible & Impossible & Impossible & Possible \\
\hline $\begin{array}{l}\text { Identify model success } \\
\text { while creating the model }\end{array}$ & Impossible & Impossible & Impossible & Possible \\
\hline Model failure rate & $2 / 12(17 \%)^{14}$ & $6 / 26(23 \%)^{19}$ & $3 / 13(23 \%)^{14}$ & $2 / 8(25 \%)$ \\
\hline 24-hour mortality & $1 / 10(10 \%)^{14}$ & $1 / 26(4 \%)^{19}$ & $1 / 10(10 \%)^{14}$ & $0 / 8(0 \%)$ \\
\hline SAH rate & $8-30 \%{ }^{14,16-18}$ & $4 / 26(15 \%)^{19}$ & $0 / 13(0 \%)^{14}$ & $0 / 8(0 \%)$ \\
\hline Reperfusion stroke model & Possible & Possible & Impossible & Impossible \\
\hline
\end{tabular}

AChA; anterior choroidal artery, ECA; external carotid artery, HTA; hypothalamic artery, MCA; middle cerebral artery, PcomA; posterior communicating artery, SAH; subarachnoid hemorrhage, VTA; ventral thalamic artery.

\section{Figures}




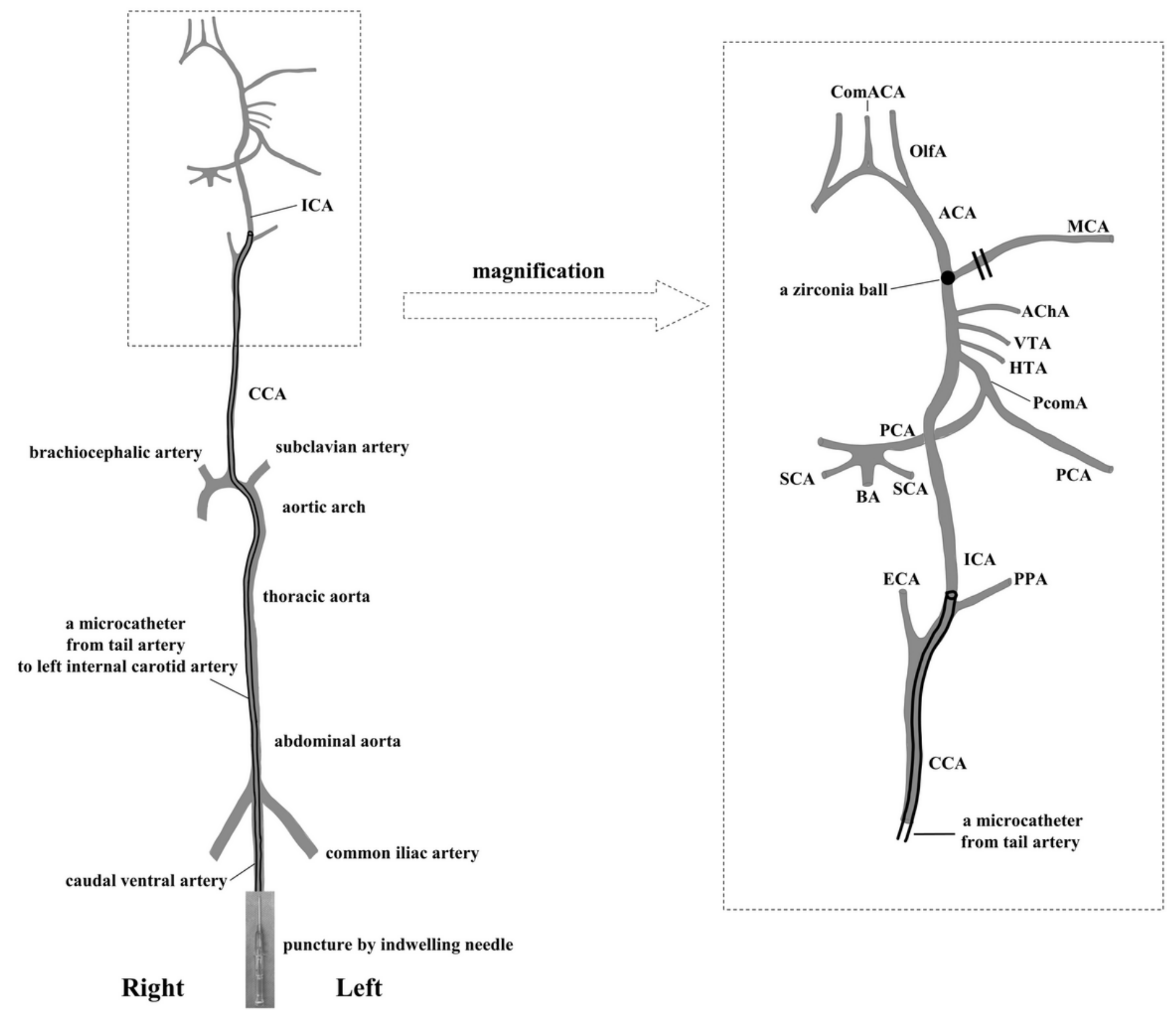

\section{Figure 1}

Schematic representation of the major rat arteries (view from the ventral) and the appropriate zirconia ball position during occlusion of the MCA. The microcatheter is inserted into ICA through caudal artery, aorta and CCA using fluoroscopic imaging. One zirconia ball is advanced in the microcatheter by slow injection and selectively occlude the ACA-MCA bifurcation. The zirconia ball block bold flow to the MCA, but not to the AChA, VTA and HTA. ACA; anterior cerebral artery, AChA; anterior choroidal artery, BA; basilar artery, CCA; common carotid artery, ComACA; common (azygos) anterior cerebral artery, ECA; external carotid artery, HTA; hypothalamic artery, ICA; internal carotid artery, MCA; middle cerebral artery, OlfA; olfactory artery, PCA; posterior cerebral artery, PcomA; posterior communicating artery, PPA; pterygopalatine artery, SCA; superior cerebellar artery, VTA; ventral thalamic artery. 


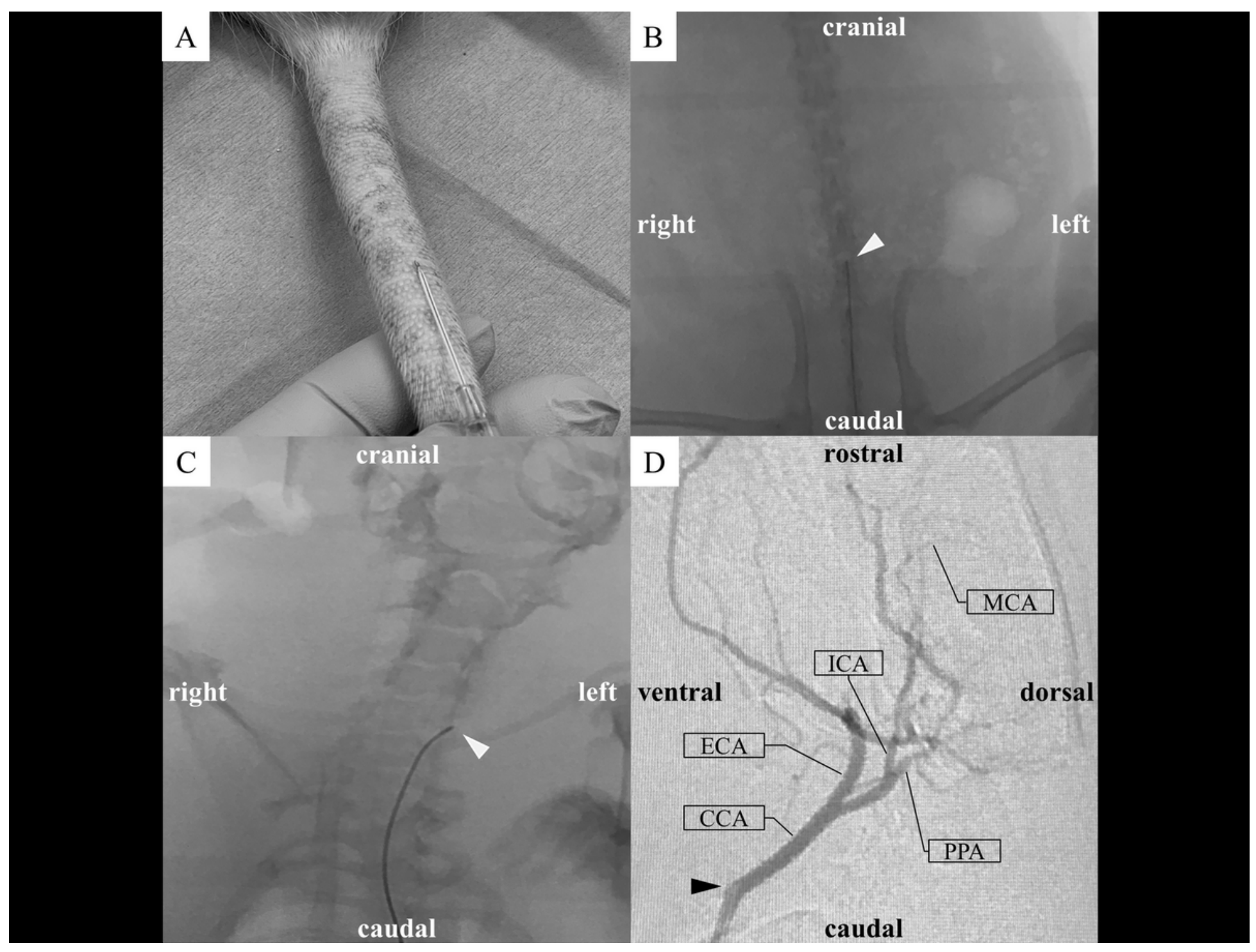

Figure 2

$<p>$ The minimally invasive intra-arterial approach under fluoroscopic guidance. A) Puncture of the caudal ventral artery in a rat. An indwelling needle was inserted through the ventral midline, approximately $5 \mathrm{~cm}$ from the root of the tail, at a sharp angle. B-C) Rat fluoroscopic images of the microcatheter and wire (arrowhead) inserted carefully through the plastic outer indwelling needle, and then inserted into the caudal ventral artery and guided into the abdominal aorta (B) and the left CCA (C). C) The C-arm was tilted at a 20\&deg; LAO angle for easy cannulation of the left CCA. D) Left CCA angiography (LAO angle =80\&deg;). The arrowhead shows the tip of the microcatheter. $\langle/ p\rangle\langle p\rangle C C A$; common carotid artery, ECA; external carotid artery, ICA; internal carotid artery, LAO; left anterior oblique, MCA; middle cerebral artery, PPA; pterygopalatine artery $</ p>$ 


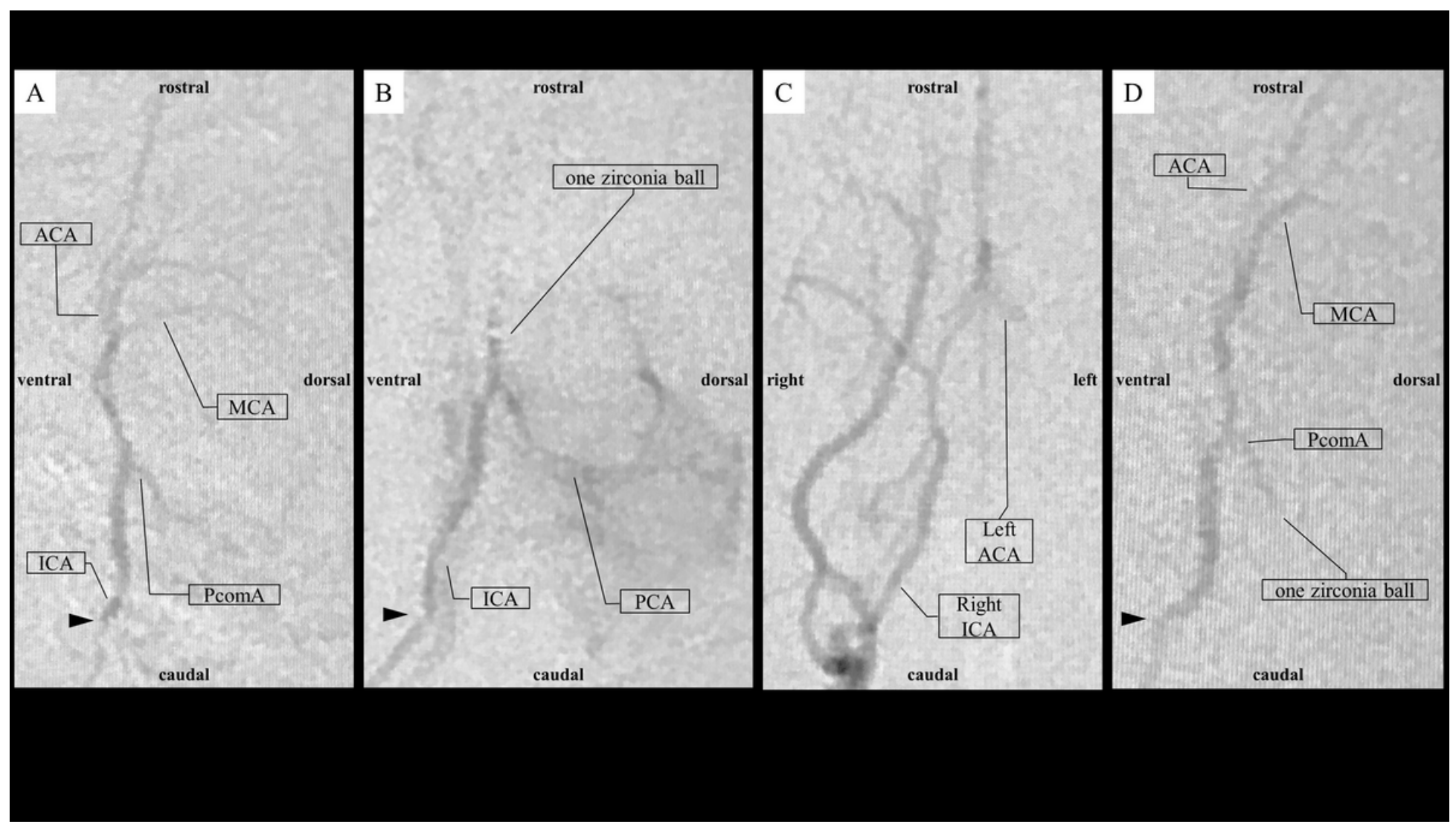

\section{Figure 3}

Angiography and embolization. The arrowhead shows the tip of the microcatheter. A) Left ICA angiography $\left(\mathrm{LAO}\right.$ angle $\left.=80^{\circ}\right)$. B) The left ACA and MCA bifurcation was selectively embolized by one zirconia ball ( $L A O$ angle $=80^{\circ}$ ). The PCA was better visualized than before embolization. C) Right CCA angiography $\left(\mathrm{LAO}\right.$ angle $\left.=0^{\circ}\right)$. The left ACA retrograde flow is maintained through the right ACA. D) The left PCA was accidentally failure embolized through the PcomA by one zirconia ball $\left(\mathrm{LAO}\right.$ angle $\left.=80^{\circ}\right)$. ACA; anterior cerebral artery, ICA; internal carotid artery, LAO; left anterior oblique, MCA; middle cerebral artery, PCA; posterior cerebral artery, PcomA; posterior communicating artery 


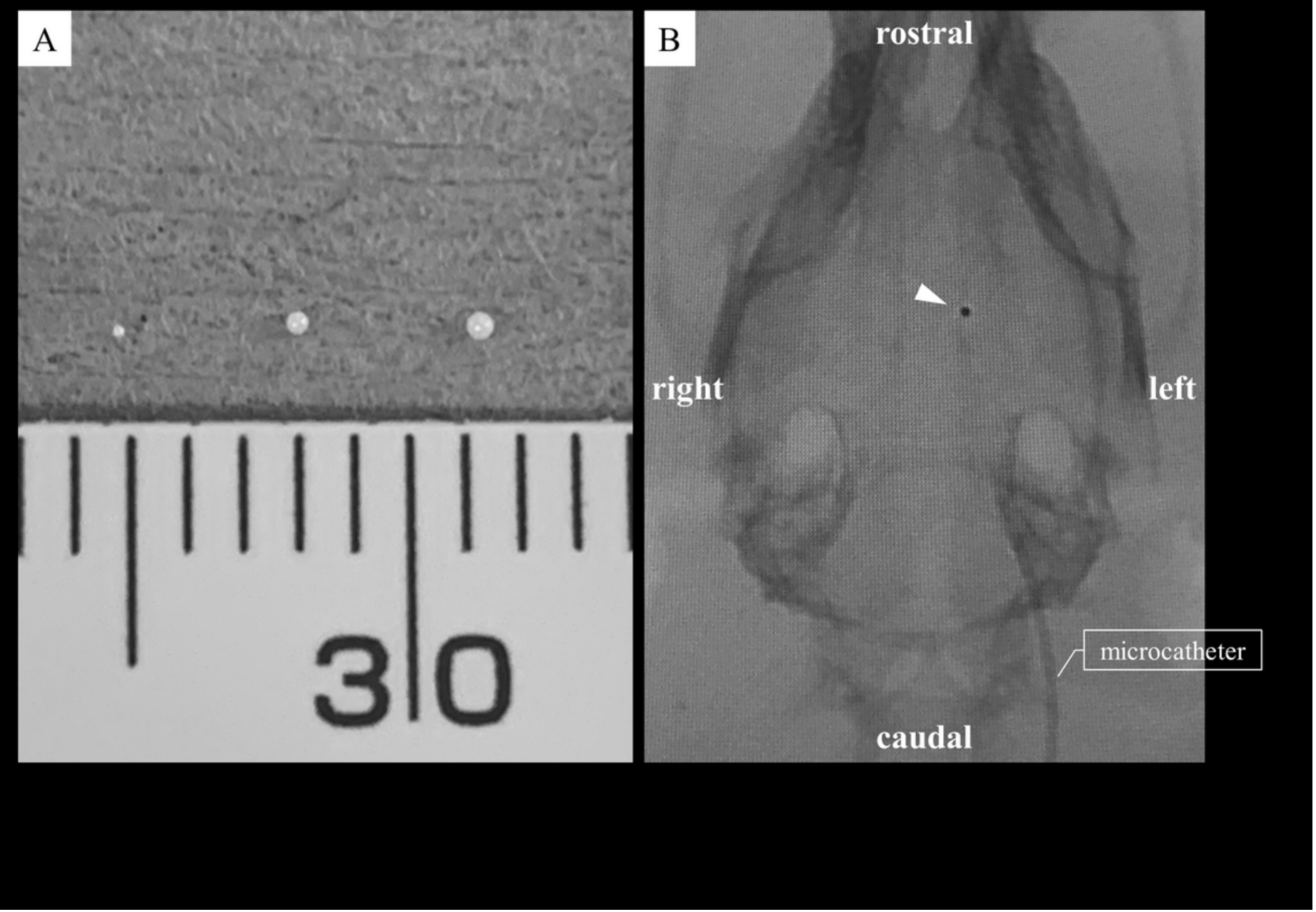

Figure 4

A) Three sizes of zirconia ball. From left to right: $0.2 \mathrm{~mm}, 0.4 \mathrm{~mm}$ and $0.5 \mathrm{~mm}$ in diameter. The zirconia ball used in the stroke model was $0.4 \mathrm{~mm}$ in diameter. B) Fluoroscopy shows the anterior cerebral arterymiddle cerebral artery bifurcation that was selectively embolized by one zirconia ball $0.4 \mathrm{~mm}$ in diameter (arrowhead). The site of embolization can be easily identified during fluoroscopy because of the high visibility of zirconia ball. 


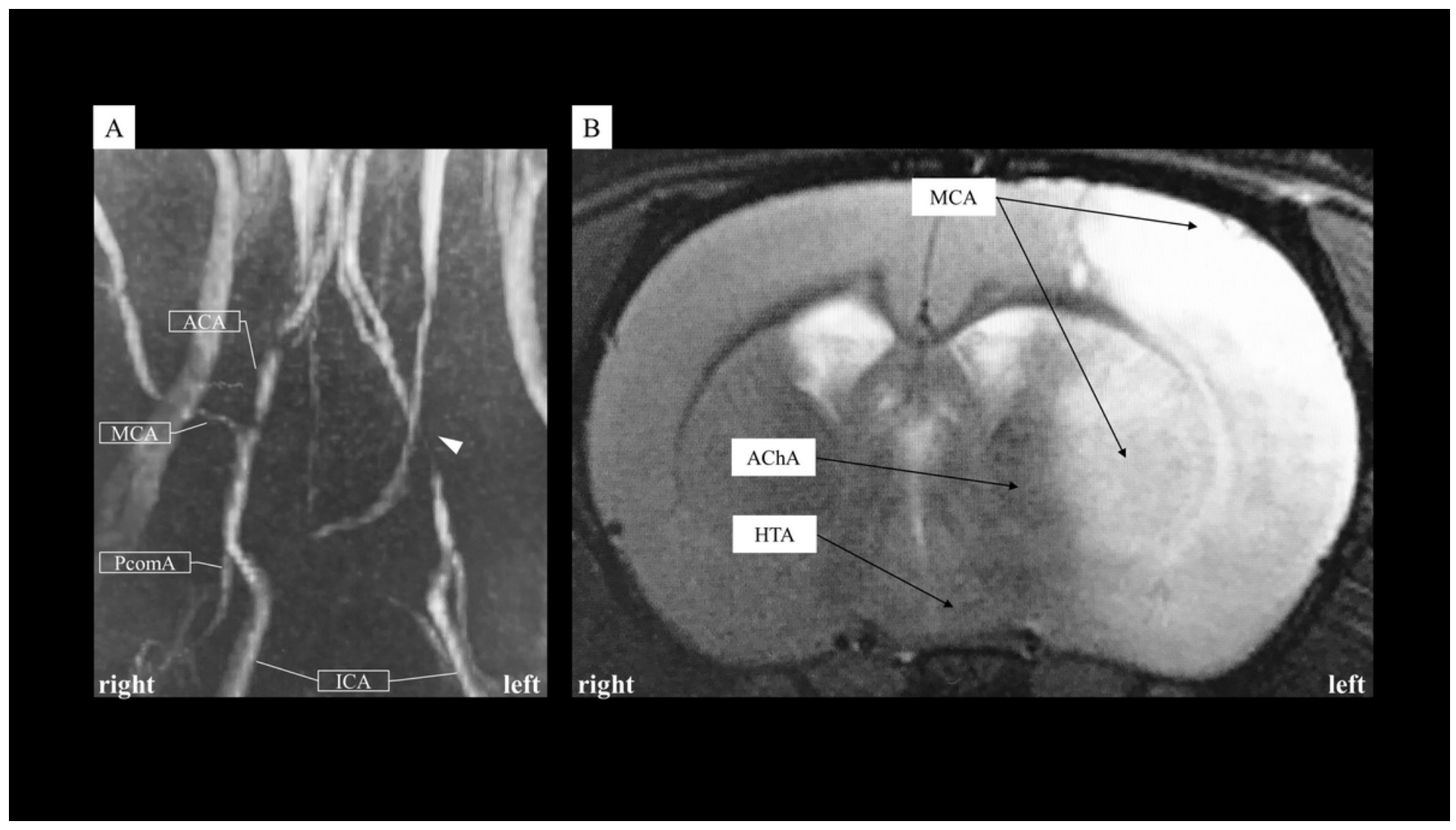

\section{Figure 5}

MRI 24 hours after the creation of the zirconia MCA occlusion model. The zirconia ball did not affect the MRI. A) MR angiography detected the impaired blood flow in the left MCA. B) T2-weighted MRI showed infarction only in the MCA perfusion area, but not in the AchA and HTA. AChA; anterior choroidal artery, HTA; hypothalamic artery, MCA; middle cerebral artery, MRl; Magnetic resonance imaging. 


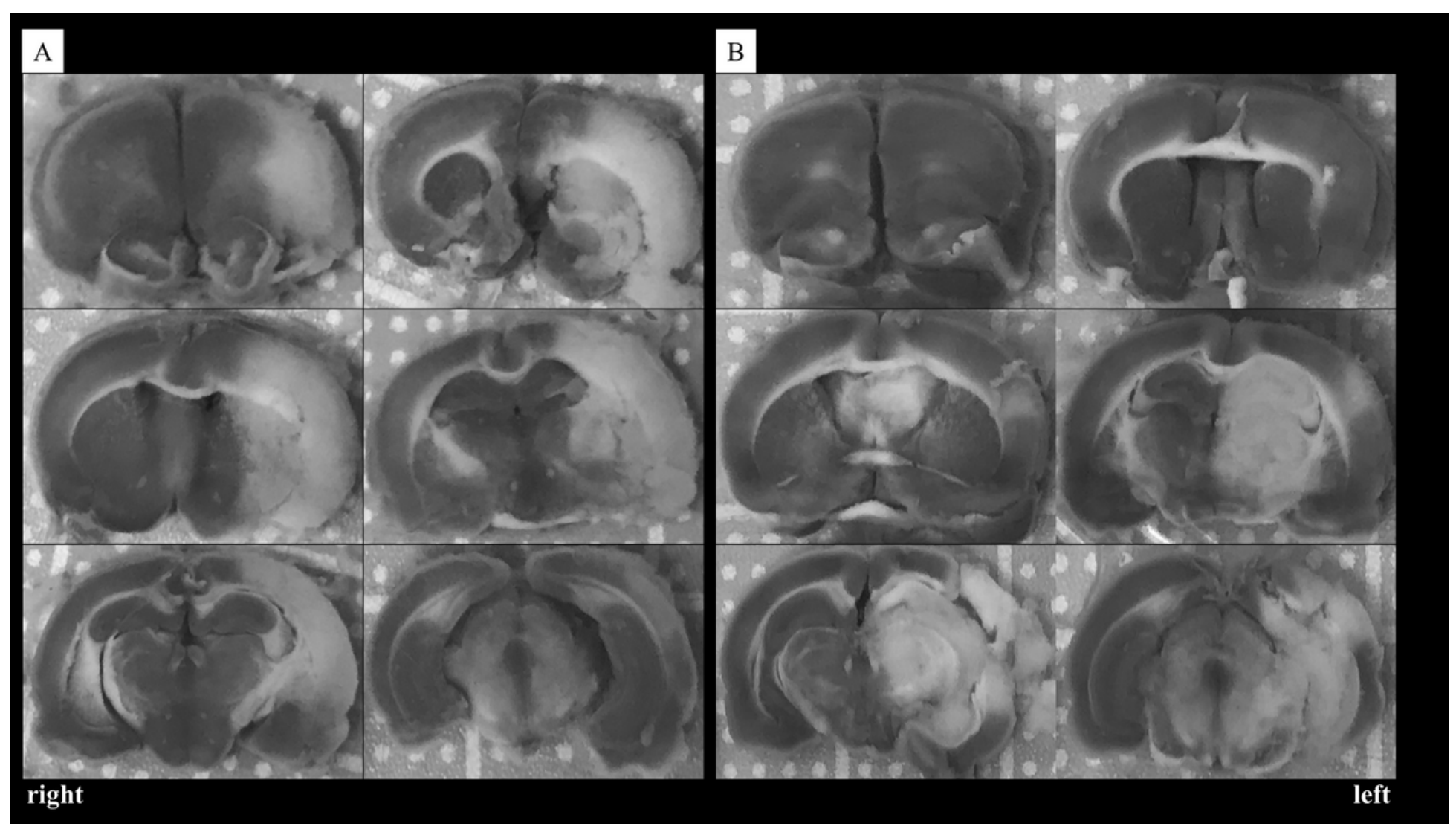

\section{Figure 6}

The brain is sliced and stained with $2 \%$ TTC solution 24 hours after the left cerebral artery occlusion. A) Brain infarction in the territory of only the MCA, but not in the AChA, VTA and HTA is clearly visible on TTC staining. B) TTC staining showed that the left PCA territory changed post-infarction. Infarction of the hippocampus and substantia nigra were observed. AChA; anterior choroidal artery, HTA; hypothalamic artery, MCA; middle cerebral artery, PCA; posterior cerebral artery, TTC: 2,3,5-triphenyltetrazolium chloride, VTA; ventral thalamic artery.

\section{Supplementary Files}

This is a list of supplementary files associated with this preprint. Click to download.

- SupplementaryVideoLegends.docx

- video1.mov

- video2.mov 